\title{
Treatment with alfacalcidol in elderly people significantly decreases the high risk of falls associated with a low creatinine clearance of $<65 \mathrm{ml} / \mathrm{min}$
}

\author{
Laurent Dukas · Erich Schacht · Ze'ev Mazor \\ Hannes B. Stähelin .
}

Received: 31 March 2003/ Accepted: 10 May 2004/Published online: 17 June 2004

(c) International Osteoporosis Foundation and National Osteoporosis Foundation 2004

\begin{abstract}
We previously observed that a creatinine clearance $(\mathrm{CrCl})$ of $<65 \mathrm{ml} / \mathrm{min}$ is a significant and independent risk factor for the number of fallers and falls in a community-dwelling elderly population and postulated that this increased risk is due to the associated significant lower D-hormone serum levels. To test our hypothesis, we investigated in a post hoc analysis of a double-blind randomized study whether treatment with alfacalcidol, a synthetic prodrug of the D-hormone, can reduce the high incidence of fallers and the high risk of falls associated with low $\mathrm{CrCl}$. Of 378 Swiss community-dwelling women $(n=191)$ and men $(n=187)$, aged 70 years and older, 191 received randomly $1 \mu \mathrm{g}$ capsules of alfacalcidol (AlphaD3: Teva), and 187 received one capsule of placebo daily. With the help of questionnaires we regularly assessed the incidence and frequency of falls. The risk of becoming a faller and the risk of falling were assessed in multivariate-controlled logistic regression models according to treatment groups and according to a $\mathrm{CrCl}$ cut-off of $65 \mathrm{ml} / \mathrm{min}$. The presented results are from ITT analyses. In participants with a $\mathrm{CrCl}$ of $<65 \mathrm{ml} / \mathrm{min}$, the 36 weeks of treatment with alfacalcidol was, compared
\end{abstract}

L. Dukas · H. B. Stähelin

Geriatric University Clinic, Kantonsspital, Basel, Switzerland

E-mail: hannes-b.staehelin@unibas.ch

Tel.: + 41-61-2652954

Fax: +41-61-2652670

E. Schacht

Metabolic Bone Disease Unit, Universitätsklinik Balgrist, Zürich, Switzerland

Z. Mazor

Bone Metabolism Unit, TEVA Pharmaceutical Industries, Jerusalem, Israel

L. Dukas $(\bowtie)$

Geriatric University Clinic, Ambulatorium Wiesendamm, Wiesendamm 22, 4057 Basel, Switzerland

E-mail: 1.d@gmx.net

Tel.: + 41-61-6312525

Fax: +41-61-6314038 with placebo, associated with a significant reduction in the number of fallers (14/72 versus $25 / 70$; OR 0.26 , $95 \%$ CI $0.08-0.80, P=0.019)$, and a significant reduction of the number of falls (16/72 versus 28/70; OR $0.29,95 \%$ CI $0.09-0.88, P=0.028)$. No such association was observed in participants with a $\mathrm{CrCl}$ of $\geq 65 \mathrm{ml} / \mathrm{min}$ (for fallers 26/120 versus 21/116; OR $0.9295 \%$ CI 0.34 $2.52, P=0.875$; for falls $32 / 120$ versus $23 / 116$; OR 0.93 95\% CI $0.34-2.54, P=0.885)$. In the placebo group frequency of falls was dependent on $\mathrm{CrCl}(P=0.006)$, whereas in the alfacalcidol treatment group frequency of falls was independent of $\mathrm{CrCl}(P=0.494)$. No cases of clinically relevant hypercalcemia were observed. In a community-dwelling population of elderly men and women with a $\mathrm{CrCl}$ of $<65 \mathrm{ml} / \mathrm{min}$, treatment with alfacalcidol can significantly and safely reduce the low $\mathrm{CrCl}$ associated increased number of fallers and the high risk of falls.

Keywords Alfacalcidol - Creatinine clearance · Elderly $\cdot$ Fallers $\cdot$ Falls

\section{Introduction}

Recently published studies [1, 2] have shown that treatment with either calcitriol in osteopenic vitamin D replete women [1] or alfacacidiol, a synthetic D-hormone pro-drug, in a population of community-dwelling elderly vitamin $\mathrm{D}$ replete men and women [2] can significantly reduce the frequency of falls $[1,2]$ and number of fallers [2]. This positive effect of calcitriol or alfacalcidol on risk of falls or number of fallers is most probably due to an increase in muscle strength $[3,4,5]$ and neuromuscular coordination [6], improved balance $[4,5,7]$ and increased functional mobility $[5,8]$ observed with increasing D-hormone serum levels or under treatment with calcitriol or alfacalcidol [7, 9].

We found that a creatinine clearance of $<65 \mathrm{ml} / \mathrm{min}$ is a significant independent new risk factor for an 
increased frequency of falls and for becoming a faller in a community-dwelling elderly population of men and women. The reduced serum creatinine clearance of $<65 \mathrm{ml} / \mathrm{min}$ was significantly associated with low D-hormone serum concentrations, and low D-hormone serum levels have been associated with an increased risk of falls or risk of becoming a faller $[9,10,11,12]$. We therefore postulated that the low $\mathrm{CrCl}$ associated risk of falls and risk of becoming a faller, which we observed in vitamin $\mathrm{D}$ replete elderly men and women, is due to the low $\mathrm{CrCl}$ associated low D-hormone serum levels. Therefore, we investigated in a post-hoc analysis in this same population of community-dwelling elderly men and women, if treatment with alfacalcidol could reduce the low creatinine clearance associated risk of falls and risk of becoming a faller.

\section{Materials and methods}

This investigation was conducted as a post-hoc analysis of the Swiss Aims Study [2]. The Aims study was a large double-blind placebo-controlled randomized study to assess the influence of a 36-week treatment with alfacalcidol ( $1 \alpha$-dihydroxycholcalcitriol, AlphaD $\mathrm{D}_{3}$; Teva Pharmaceutical Industries, Jerusalem, Israel), a D-hormone pro-drug, on frequency of fallers and falls in community-dwelling elderly men and women, 70 years old and older. The study has previously been described in detail [2]. Of the 378 participants (191 women and 187 men), aged 70 years old and older, 191 randomly received $1 \mu \mathrm{g}$ capsules of alfacalcidol, and 187 received one capsule of placebo daily. Falls were assessed every 12 weeks after the baseline visit for a total of 36 weeks. The procedure for assessment of falls has been previously described in detail [2]. The dietary calcium and vitamin $\mathrm{D}$ intake was estimated by using a modified food frequency questionnaire [13], which also gave information on some demographic, lifestyle and nutritional parameters. Calcium was not supplied, none of the participants was receiving physical therapy or training programs at study entry, and no attempt was made to alter subjects' diet or physical activity during the study. In all subjects, blood samples were drawn for measurement of serum calcium, phosphate, creatinine, calcidiol, D-hormone and iPTH. Calcidiol, D-hormone and iPTH serum levels were measured by radioimmunoassay (Nichols). In this manuscript, we use the terminology of D-hormone for 1,25-dihydroxycalcitriol to clearly differentiate between vitamin $\mathrm{D}$ and the hormonal activity of D-hormone. The protocol of this study was approved by the Ethical Review Board of the University of Basel, and all participants provided written informed consent. The Data, Safety and Monitoring Board established by GWD Consult Germany (Safety and Monitoring Board: GWD Consult, Research Contract, Postfach 1210, 63152 Mülheim/Main, Germany), reviewed the conduct of the study.

\section{Statistical analyses}

Comparisons of means were performed by multivariate adjusted analyses of variance [14]. The main follow-up multivariate analysis compared the number of fallers and falls in the two treatment groups according to a previously determined cut-off value for creatinine clearance set at $65 \mathrm{ml} / \mathrm{min}$. Those factors that reached significance level of $P<0.1$ in a screening regression model were entered into a logistic regression model. Multivariate difference in number of fallers and falls between treatment groups are given as odds ratio (OR) with $95 \%$ confidence intervals (CI). According to our previously published results [2] on the effect of total daily calcium intake on the treatment with alfacalcidol, we also performed subgroup analyses according to creatinine clearance and total daily calcium intake; even so, our study was not designed and did not have the power to detect significant differences in such small subgroups. The results presented are from multivariate-controlled ITT analyses. A 5\% significance level was maintained throughout these analyses, and all tests were two-sided.

The statistical analyses were conducted using the SAS statistical software package, version 8.2 , by the SAS Institute Inc., Cary N.C., USA, licensed to the University of Basel, Switzerland.

\section{Results}

\section{General}

The two treatment groups were comparable at baseline. A significant difference was found only for the use of phenprocoumon. Average dietary calcium intake was generally low $[$ mean $( \pm \mathrm{SD})$ daily intake in $\mathrm{mg}$ : $512 \pm 197$, respectively $521 \pm 147]$. The proportion of men to women was the same in each treatment group and major comorbid conditions were equally distributed between treatment groups (data not shown). Also, in participants with a creatinine clearance of $\geq 65 \mathrm{ml} / \mathrm{min}$, the two treatment groups were comparable at baseline, with a comparable gender distribution. A significant difference was found only for the use of aspirin, and average dietary calcium intake was also generally low (mean \pm SD daily intake in $\mathrm{mg}$ : $530 \pm 167$, respectively $514 \pm 159)$. Comorbid conditions were equally distributed between treatment groups (data not shown).

Compared with participants with a creatinine clearance of $\geq 65 \mathrm{ml} / \mathrm{min}$, participants with a creatinine clearance of $<65 \mathrm{ml} / \mathrm{min}$ were multivariate controlled significantly older $(P<0.0001)$, and had multivariate controlled significant lower BMI $(P<0.0001)$, lower D-hormone serum levels $(P=0.010)$ and lower bone density measured as $T$-score with ultrasound of the calcaneus $(P=0.023)$. Furthermore, participants with a creatinine clearance of $<65 \mathrm{ml} / \mathrm{min}$ were significantly more likely to be female $(P=0.030)$ and to live alone $(P=0.042)$, were less likely to be diabetic $(P=0.048)$, 
were significantly more likely to be under a daily medication with digoxin $(P=0.032)$, diuretics $(P=0.005)$ and alternative medicine $(P=0.018)$ and were less physically active $(P=0.015)$ (Table 1$)$. For all other variables we found no significant differences between groups according to creatinine clearance.

\section{Follow-up}

Of the 378 participants enrolled, $321[84.3 \% / 158$ women $(82.7 \%)$ and 163 men $(87.2 \%)]$ completed the study, with no difference in completion rate between treatment groups (placebo $83.3 \%$ versus alfacalcidol $86.5 \%, P=0.649)$ During the study there were two deaths, both in the group with a $\mathrm{CrCl}<65 \mathrm{ml} / \mathrm{min}$ : one man on placebo, with long-standing heart disease, died from myocardial infarction, and one man on alfacalcidol after a bout of influenza died from pneumonia and subsequent hepatitis ( $P=0.982$, for the difference in death rates according to treatment group).

\section{Fallers and falls (Table 2, Fig. 1 and 2)}

The multivariate analyses included predictors which have been previously shown to be associated with increased risk of falling, or variables which were significantly different between treatment groups. These variables were: age, gender, body mass index (BMI), creatinine clearance $(\mathrm{ml} / \mathrm{min})$, number of falls in previous 3 months, physical activity (sport yes/no), Charlson comorbidity index, number of medications at baseline, dietary calcium intake, heart rate at baseline $(<80$ beats $/ \mathrm{min}$ versus $\geq 80$ beats $/ \mathrm{min})$, D-hormone $(\mathrm{pg} / \mathrm{ml})$, calcidiol $(\mathrm{ng} / \mathrm{ml})$, iPTH $(\mathrm{pg} / \mathrm{ml})$, creatinine $(\mu \mathrm{mol} / \mathrm{l})$ and albumin $(\mathrm{g} / \mathrm{l})$ serum levels at baseline, changes in D-hormone, calcidiol and iPTH serum levels over time

Table 1 Comparison of the

characteristics of the study participants with a Creatinine clearance of $<65 \mathrm{ml} / \mathrm{min}$ vs $\geq 65 \mathrm{ml} / \mathrm{min}$. BMI body mass index, $i P T H$ intact parathormone, QUS quantitative ultrasound (calcaneus), controlled for gender

\begin{tabular}{llll}
\hline Categories & $\begin{array}{l}\mathrm{CrCl}<65 \mathrm{ml} / \mathrm{min} \\
(n=142)\end{array}$ & $\begin{array}{l}\mathrm{CrCl} \geq 65 \mathrm{ml} / \mathrm{min} \\
(n=236)\end{array}$ & $P$-value \\
\hline Gender (number), male/female & $60 / 82$ & $127 / 109$ & 0.030 \\
Age (mean $\pm \mathrm{SD}$ ) years & $76.6 \pm 4.5$ & $74.0 \pm 3.8$ & $<0.0001$ \\
BMI (mean $\pm \mathrm{SD}) \mathrm{kg} / \mathrm{m}^{2}$ & $24.6 \pm 3.0$ & $27.5 \pm 3.9$ & $<0.0001$ \\
Laboratory (mean $\pm S D)$ & & & \\
iPTH pg/ml & $40.6 \pm 27.5$ & $36.4 \pm 15.4$ & 0.057 \\
D-hormone pg/ml & $37.6 \pm 11.9$ & $40.7 \pm 11.2$ & 0.010 \\
Calcidiol ng/ml & $28.5 \pm 11.8$ & $29.8 \pm 15.8$ & 0.422 \\
Albumin g/l & $42.5 \pm 2.8$ & $42.1 \pm 2.9$ & 0.150 \\
Drug intake (in \%) & & & \\
Use of alternative medicine & $7.2 \%$ & $2.1 \%$ & 0.018 \\
Use of digoxin & $4.9 \%$ & $1.3 \%$ & 0.032 \\
Use of diuretics & $23.3 \%$ & $17.8 \%$ & 0.005 \\
Other variables (in \% or mean $\pm S D)$ & & & \\
Living alone & $38.0 \%$ & $28.0 \%$ & 0.042 \\
Number of diabetics & $1.4 \%$ & $5.5 \%$ & 0.048 \\
History of falls in previous 3 months & $12.7 \%$ & $10.7 \%$ & 0.453 \\
before study entry & & & 0.015 \\
Regular physical activity (yes) & $33.1 \%$ & $45.7 \%$ & 0.751 \\
Daily dietary calcium intake (mg) & $516 \pm 172$ & $522 \pm 163$ & 0.023 \\
Bone density QUS $(T$-score) & $-1.08 \pm 1.58$ & $-0.64 \pm 1.39$ & 0.518 \\
Timed up and go test (s) & $7.0 \pm 1.8$ & $6.9 \pm 1.7$ & \\
\hline
\end{tabular}

Table 2 Effect of alfacalcidol in elderly men and women on number of fallers and falls according to creatinine clearance: cut-off $<65 \mathrm{ml} /$ $\mathrm{min} / \geq 65 \mathrm{ml} / \mathrm{min}$

\begin{tabular}{llllr}
\hline Creatinine clearance & Parameter & $\begin{array}{l}\text { Multivariate } \\
\text { Adjusted OR (95\% CI) }\end{array}$ & $\begin{array}{l}\text { Number of fallers/falls } \\
\text { Alfacalcidol vs Placebo }\end{array}$ \\
\hline$<65 \mathrm{ml} / \mathrm{min}$ & Fallers & $0.26(0.08-0.80)$ & $14 / 25$ & $0.019^{\mathrm{a}}$ \\
& Falls & $0.29(0.09-0.88)$ & $16 / 28$ & $0.028^{\mathrm{a}}$ \\
& Fallers & $0.92(0.34-2.52)$ & $32 / 21$ & $0.875^{\mathrm{a}}$ \\
& Falls & $0.93(0.34-2.54)$ & $32 / 23$ & $0.885^{\mathrm{a}}$ \\
\hline
\end{tabular}

${ }^{a}$ Controlled for age, gender, BMI, previous falls, use of diuretics, albumin serum levels, calcidiol, D-hormone and iPTH serum levels at baseline and during follow-up, total daily calcium intake and coffee consumption, use of diuretics, marcoumar, aspirin, estrogens and multivitamins, number of co-morbidities and physical activity 
Fig. 1 Number of fallers over time according to treatment groups

Fig. 2 Number of falls over time according to treatment groups

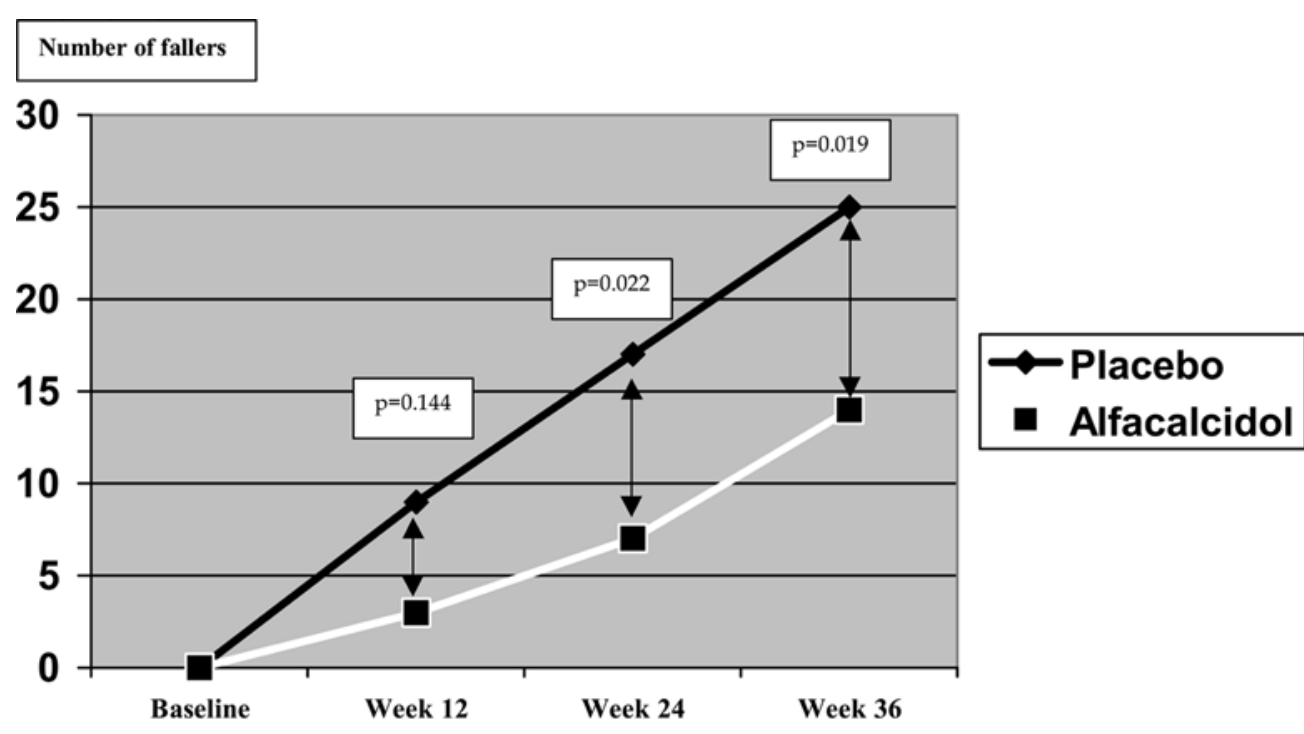

Observation Time

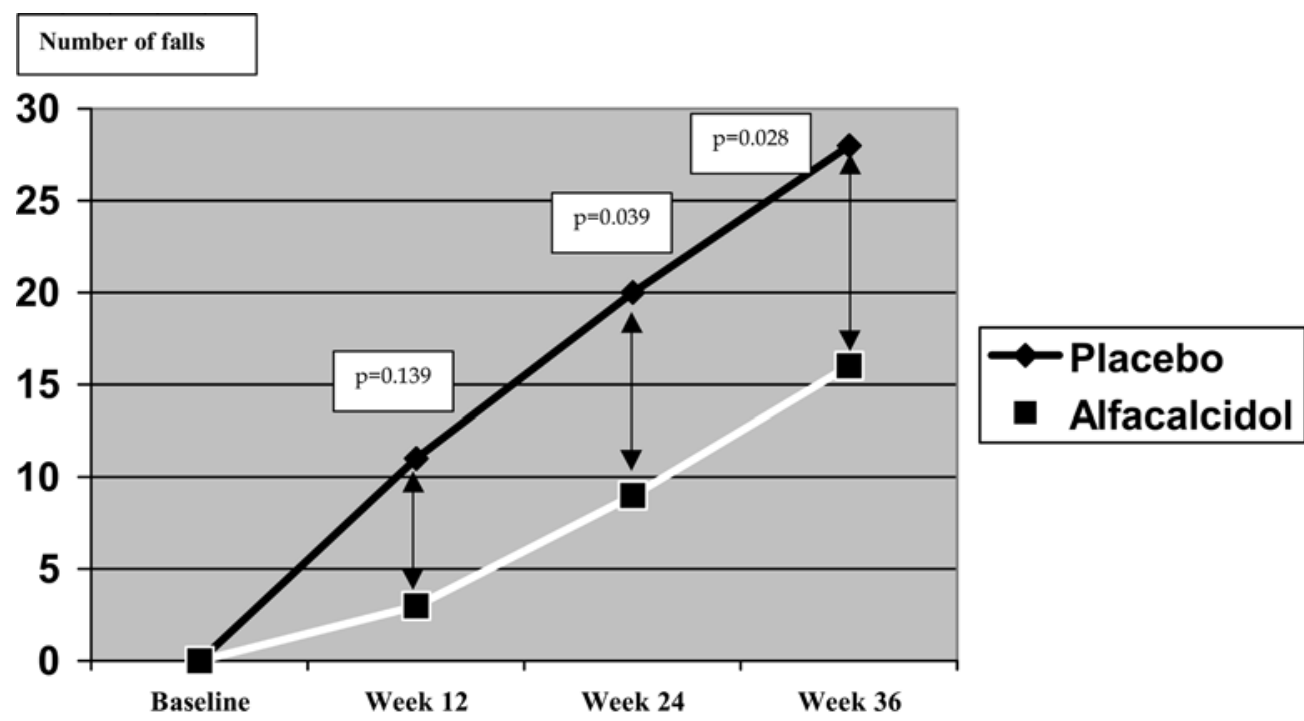

Observation Time in percent, coffee intake, use of estrogen, phenprocoumon and aspirin.

In the multivariate adjusted logistic regression analyses, treatment with alfacalcidol over 36 weeks was, compared with placebo, in participants with a creatinine clearance below $65 \mathrm{ml} / \mathrm{min}$ associated with a significant reduction in number of fallers $(14 / 72$ versus $25 / 70$; OR $0.26,95 \%$ CI $0.08-0.80, P=0.019)$, and a significant reduction in the number of falls $(16 / 72$ versus $28 / 70$; OR $0.29,95 \%$ CI $0.09-0.88, P=0.028$ ) (Table 2). No such association was observed in participants with a $\mathrm{CrCl}$ of $\geq 65 \mathrm{ml} / \mathrm{min}$ (for fallers $26 / 120$ versus $21 / 116$; OR 0.92 $95 \%$ CI $0.34-2.52, P=0.875$; for falls $32 / 120$ versus 23 / 116; OR 0.93 95\% CI 0.34-2.54, $P=0.885$ ) (Table 2).
The difference in the reduction of number of fallers and number of falls between treatment groups became already significant after 24 weeks of intervention for number of fallers and for number of falls (alfacalcidol versus placebo week 24: number of fallers 7 versus 17 , OR $0.1895 \%$ CI $0.014-0.078, P=0.022$; number of falls 9 versus 20, OR $0.2295 \%$ CI $0.05-0.93, P=0.039$ ) (Fig. 1 and 2).

In subgroup analyses of participants with creatinine clearance of $<65 \mathrm{ml} / \mathrm{min}$ according to the median of total daily calcium intake, alfacalcidol had a non-significant greater effect in participants who were stratified in the group above the median of total daily calcium intake compared with participants stratified into the 
group below the median daily of total daily calcium intake (OR 0.30 95\% CI $0.07-1.25$ versus OR $0.6695 \%$ CI $0.18-2.38$ ) (data not shown). In the alfacalcidol treatment group, frequency of falls was independent from $\mathrm{CrCl}(P=0.494)$ (data not shown).

\section{Adverse events and side effects}

During the 36 weeks of intervention, we observed six cases [one case in the placebo $(\mathrm{CrCl} \geq 65 \mathrm{ml} / \mathrm{min})$ group and five cases in the alfacalcidol group (one with a $\mathrm{CrCl}$ $\geq 65 \mathrm{ml} / \mathrm{min}$ and four with a $\mathrm{CrCl}<65 \mathrm{ml} / \mathrm{min}$ )] of slight transient hypercalcemia (one measurement of serum calcium above normal with subsequent (1 week later) control measurement within normal ranges), the highest value measured being $2.73 \mathrm{mmol} / 1$ (normal range 2.10 $2.65 \mathrm{mmol} / \mathrm{l})$. This slight transient increase in serum calcium did not lead to a modification of the dose of alfacalcidol. Two participants in the alfacalcidol group, one with a $\mathrm{CrCl} \geq 65 \mathrm{ml} / \mathrm{min}$ and one with a $\mathrm{CrCl}$ $<65 \mathrm{ml} / \mathrm{min}$ ), presented after 12 weeks of intervention continuously till the end of the study elevated calcium serum levels (highest value $3.00 \mathrm{mmol} / \mathrm{l}$ ), without clinical symptoms. One of these participants admitted at the end of the study to having taken supplementary calcium (1000 mg daily). The difference in the incidence of hypercalcemia between study groups and according to $\mathrm{CrCl}$ was not significant $(P=0.0621$, respectively $P=0.260$ ). Furthermore, we observed no differences in the frequency of major diseases occurring during the 36 weeks between treatment groups and no cases of serious adverse events attributable to alfacalcidol treatment. Frequency of reported side effects was equally distributed between treatment groups ( 82 cases in placebo versus 75 cases in alfacalcidol, $P=0.850)$ and independent from creatinine clearance $(P=0.878)$. The most common side effects were itching (placebo treatment group: 23 cases/alfacalcidol treatment group: 22 cases) and skin eruption (placebo treatment group: 11 cases/ alfacalcidol treatment groups 15 cases).

\section{Discussion}

We are the first to describe a low creatinine clearance associated risk for falls and risk of becoming a faller, which we attributed to the significantly lower D-hormone serum levels observed with a creatinine clearance below $65 \mathrm{ml} / \mathrm{min}$. Therefore, we are also the first to have studied the effect of treatment with alfacalcidol on low creatinine clearance associated risk of falls. We observed in community-dwelling elderly men and women that treatment with alfacalcidol significantly reduced the high risk of falls and risk of becoming a faller associated with a creatinine clearance $<65 \mathrm{ml} / \mathrm{min}$. The treatment effect of alfacalcidol on number of fallers and number of falls already became significant after 24 weeks of treatment with alfacalcidol. In participants with a $\mathrm{CrCl}<65 \mathrm{ml} /$ min, alfacalcidol reduced the frequency of fallers and falls to the frequency of fallers and falls observed in participants with a $\mathrm{CrCl} \geq 65 \mathrm{ml} / \mathrm{min}$, for whom we found no difference in fall and faller frequency between treatment groups.

D-hormone has a scientifically established effect on muscle strength, balance and functional mobility $[3,4,5$, $6,7,8,9]$, and the effect of D-hormone treatment (alfacalcidol or calcitriol) on risk of falls has been proven in several studies $[1,2]$. The low D-hormone serum levels observed with a creatinine clearance of $<65 \mathrm{ml} / \mathrm{min}$ may also be one reason for the increased osteopenia and osteoporosis observed with low creatinine clearance by other authors $[15,16]$. Our result enhances the theory suggested by other studies $[4,5,6,7$, $17,18,19,20,21,22]$ that $\mathrm{D}$-hormone is an independent risk factor for falls $[1,2,5,23]$, and that D-hormone is directly involved in the causal pathogenic pathway of decreased muscle strength related falls [9, 23, 24]. It has been recently confirmed in VDR gene deleted mice that the absence of VDR causes muscle abnormality independently of secondary metabolic changes, e.g. hypocalcemia or hyperparathyroidism, and that treatment with D-hormone counterbalances the abnormalities in myoblastic cells and is necessary for optimal muscle differentiation [25]. We therefore conclude that our previously observed approximately 4 times increased risk of falls observed in participants with a low creatinine clearance is due to a creatinine clearance dependent decrease in D-hormone serum levels. Therefore treatment options for patients at risk for falls should clearly differentiate between nutritive vitamin D deficiency and metabolic low D-hormone syndrome [18, 19, 20, 21].

We found an increased treatment effect of alfacalcidol on frequency of fallers and falls in participants with a $\mathrm{CrCl}<65 \mathrm{ml} / \mathrm{min}$ and a total daily calcium intake above the median of $512 \mathrm{mg}$ compared with participants with a $\mathrm{CrCl}<65 \mathrm{ml} / \mathrm{min}$ and daily total calcium intake below $512 \mathrm{mg}$. The influence of calcium intake on the treatment effect of alfacalcidol on frequency of falls and fallers was described by us previously [2]; however, in participants with a $\mathrm{CrCl}<65 \mathrm{ml} / \mathrm{min}$, the influence of calcium intake on the treatment effect of alfacalcidol was not significant. This result may be due to the fact that our study was not designed for power analyses in small subgroups.

Our study has several limitations. The results come from post hoc analyses. The participants were Caucasian community-dwelling men and women over the age of 70 years, so our findings are not generalizable to a younger population, institutionalized elderly or to men and women of other races. Since our analyses were performed in participants who received placebo during 36 weeks, we cannot rule out an influence in either direction of placebo on risk of falls. Assessment of risk factors for falls as well as the large majority of incidence of falls during the study was based only on the participant's own report. Finally, we cannot exclude uncontrolled confounding. 
In conclusion a low creatinine clearance of $<65 \mathrm{ml} /$ min associated increased risk of becoming a faller and increased risk for falls can significantly be treated with alfacalcidol. Our result confirms our hypothesis that the low creatinine clearance of $<65 \mathrm{ml} / \mathrm{min}$. associated risk of becoming a faller and for falls is due to low creatinine clearance associated low D-hormone.

Acknowledgements We are indebted to the participants and all our study co-workers; to the team from the laboratory of Rheumatology at the Felix-Platter Spital, Basel, Switzerland as well as to the team of the Hospital Pharmacy of the Kantonsspital, Basel, Switzerland; to Professor John Orav (Division of Clinical Epidemiology, Brigham and Women's Hospital, Boston, USA) for statistical consultation and advice; to Dr. A. Monsch for advice and encouragement in the preparation of the study protocol and study conduct. The study was supported by the following institutions: TEVA Pharmaceuticals Industries Ltd, Israel and a scientific grant from the University Hospital Basel, Switzerland.

\section{References}

1. Gallagher JC, Fowler SE, Detter JR et al. (2001) Combination treatment with estrogen and calcitriol in the prevention of agerelated bone loss. J Clin Endocrinol Metab 86:3618-3628

2. Dukas L, Bischoff HA, Lindpaintner LS, Schacht E, BirknerBinder D. Thalmann B, Stähelin HB (2004) Alfacalcidol reduces the number of fallers in a community-dwelling elderly population with a minimum calcium intake of $500 \mathrm{mg}$ daily. J Am Geriatr Soc 52:230-236

3. Peacock M, Heyburn P (1977) Effect of vitamin $D_{3}$ metabolites on proximal muscle weakness. Calcif Tissue Res 24:R20-23

4. Bischoff HA, Stähelin HB, Urscheler N et al. (1999) Muscle strength in the elderly: its relation to vitamin D metabolites. Arch Phys Med Rehabil 80:54-58

5. Verhaar HJJ, Samson MM, Jansen PAF et al (2000) Muscle strength, functional mobility and vitamin D in older women. Aging Clin Exp Res 12:455-460

6. Dhesi JK, Bearne LM, Monitz C, Hurley MV, Jackson SHD, Swift CG, Allain TJ (2002) Neuromuscular and psychomotor function in elderly subjects who fall and the relationship with vitamin D status. J Bone Miner Res 17:891-897

7. Koike T, Okawa T, Wada M, Kita T, Takaoka K (2003) Effects of a long-term alfacalcidol or calcitonin administration on body sway in Japanese elderly women. J Bone Miner Res 18:S168

8. Dukas L, Schacht E Bischoff HA (2003) Better functional mobility in community dwelling elderly is related to
D-hormone and a minimal calcium intake of more than $512 \mathrm{mg}$ /day. Osteoporos Int 14:S34

9. Tinetti ME, Williams CS (1998) The effect of falls and fall injuries on functioning in community-dwelling older persons. J Gerontol A Biol Sci Med Sci 53:M112-M119

10. Tinetti ME (2003) Preventing falls in elderly persons. N Engl J Med 348:42-49

11. Nevitt MC, Cummings SR, Hudes ES (1989) Risk factors for recurrent nonsyncopal falls: a prospective study. JAMA 261:2663-2668

12. Sattin RW (1992) Falls among older persons: a public health perspective Annu Rev Public Health 13:489-508

13. Willet W (1998) 1980 Nurses' health study dietary questionnaire. In Nutritional epidemiology, 2nd edn. Oxford University Press, Oxford

14. Winer BJ (1971) Statistical principles in experimental design, 2nd edn. McGraw-Hill, New York, p 514

15. Poor G, Atkinson EJ, O'Fallon WM et al. (1995) Predictors of hip fracture in elderly men. J Bone Miner Res 10:1900-1907

16. K/DOQI (2003) practice guidelines for bone metabolism and disease in chronic kidney disease Am J Kidney Dis 42:S7-S9

17. Epstein S, Bryce G, Hinman JW et al. (1986) The influence of age on bone mineral regulating hormones. Bone 7:421-425

18. Tsai KS, Heath H III, Kumar R et al. (1984) Impaired vitamin D metabolism with aging in women: possible role in pathogenesis of senile osteoporosis. J Clin Invest 73:1668-1672

19. Dukas L, Bischoff HA, Schacht E et al. (2002) Normal 25(OH) vitamin D serum levels do not exclude D-hormone deficiency in community-dwelling elderly. Osteoporos Int 13:S35

20. Slovik DM, Adams JS, Neer RM et al. (1981) Deficient production of 1,25-dihydroxyvitamin $\mathrm{D}$ in elderly osteoporotic patients. N Engl J Med 305:372-374

21. Zofkovà I, Kancheva RL, Bendlovà B (1997) Effect of $1,25(\mathrm{OH})_{2}$ vitamin $\mathrm{D}_{3}$ on circulating insulin-like growth factorI and $\beta_{2}$ microglobulin in patients with osteoporosis. Calcif Tissue Res 60:236-239

22. Cappola AR, Xue Q L, Ferrucci L, Guralnik JM, Volpato S, Fried LP (2003) Insulin-like growth factor I and interleukin-6 contribute synergistically to disability and mortality in older women. J Clin Endocrinol Metab 88:2019-2025

23. Stein MS, Wark JD, Scherer SC, Walton SL, Chick P, Di Carlantonio M, Zajac JD, Flicker L (1999) Falls related to vitamin $\mathrm{D}$ and parathyroid hormone in Australian nursing home and hostel. J Am Geriatr Soc 47:1195-1201

24. Boland R (1986) Role of vitamin D in skeletal muscle function. Endocr Rev 784:434-448

25. Endo I, Inoue D, Mitsui T, Umaki, Y, Akaike M, Yoshizawa T, Kato S, Matsumoto T (2003) Deletion of vitamin D receptor gene in mice results in abnormal skeletal muscle development with deregulated expression of myoregulatory transcription factors. Endocrinology 144:5138-5144 